\title{
Approximation Schemes for Multi-Budgeted Independence Systems ${ }^{\star}$
}

\author{
Fabrizio Grandoni ${ }^{1}$ and Rico Zenklusen ${ }^{2 \star \star}$ \\ 1 Computer Science Department, University of Rome Tor Vergata, \\ grandoni@disp.uniroma2.it \\ 2 Department of Mathematics, EPFL, \\ rico.zenklusen@epfl.ch
}

\begin{abstract}
A natural way to deal with multiple, partially conflicting objectives is turning all the objectives but one into budget constraints. Some classical optimization problems, such as spanning tree and forest, shortest path, (perfect) matching, independent set (basis) in a matroid or in the intersection of two matroids, become NP-hard even with one budget constraint. Still, for most of these problems efficient deterministic and randomized approximation schemes are known. For two or more budgets, typically only multi-criteria approximation schemes are available, which return slightly infeasible solutions. Not much is known however for strict budget constraints: filling this gap is the main goal of this paper. It is not hard to see that the above-mentioned problems whose solution sets do not correspond to independence systems are inapproximable already for two budget constraints. For the remaining problems, we present approximation schemes for a constant number $k$ of budget constraints using a variety of techniques: i) we present a simple and powerful mechanism to transform multi-criteria approximation schemes into pure approximation schemes. This leads to deterministic and randomized approximation schemes for various of the above-mentioned problems; ii) we show that points in low-dimensional faces of any matroid polytope are almost integral, an interesting result on its own. This gives a deterministic approximation scheme for $k$-budgeted matroid independent set; iii) we present a deterministic approximation scheme for 2-budgeted matching. The backbone of this result is a purely topological property of curves in $\mathbb{R}^{2}$.
\end{abstract}

\section{Introduction}

In many applications, one has to compromise between several, partially conflicting goals. Multi-Objective Optimization is a broad area of study in Operations Research, Economics and Computer Science (see [8,22] and references therein). A variety of approaches have been employed to formulate such problems. Here we adopt the Multi-Budgeted Optimization approach [22]: we cast one of the goals

* Partially developed while the first author was visiting EPFL.

** Partially supported by the Swiss National Science Foundation, grant number: PBEZP2-129524. 
as the objective function, and the others as budget constraints. More precisely, we are given a (finite) set $\mathcal{F}$ of solutions for the problem, where each solution is a subset $S$ of elements from a given universe $E$ (e.g., the edges of a graph). We are also given a weight function $\mathrm{w}: \mathcal{F} \rightarrow \mathbb{Q}_{+}$and a set of $k=O(1)^{3}$ length functions $\ell_{i}: \mathcal{F} \rightarrow \mathbb{Q}_{+}, 1 \leq i \leq k$, that assign a weight $\mathrm{w}(S):=\sum_{e \in S} \mathrm{w}(e)$ and an $i$ th-length $\ell_{i}(S):=\sum_{e \in S} \ell_{i}(e), 1 \leq i \leq k$, to every candidate solution $S$. For each length function $\ell_{i}$, there is a budget $\mathrm{L}_{i} \in \mathbb{Q}_{+}$. The multi-budgeted optimization problem can then be formulated as follows:

maximize/minimize w $(S)$ subject to $S \in \mathcal{F}, \ell_{i}(S) \leq \mathrm{L}_{i}, 1 \leq i \leq k$.

We next use $O P T$ to denote an optimum solution.

Following the literature on the topic, we focused on the set of problems below:

- $k$-BUdGeted (PERFECT) MATChing: $\mathcal{F}$ is given by the (perfect) matchings of an undirected graph $G=(V, E)$.

- $k$-BUdGeted SPANNING TREE (FOREST): $\mathcal{F}$ is given by the spanning trees (forests) of $G$.

- $k$-BUdgeted SHORTEST PATH: $\mathcal{F}$ is given by the paths connecting two given nodes $s$ and $t$ in $G$.

- $k$-Budgeted MATRoid Independent SET (BASIS): $\mathcal{F}$ is given by the independent sets (bases) of a matroid $M=(E, \mathcal{I})^{4}$.

- $k$-Budgeted MATRoid intersection indePEndent Set (BASIS): $\mathcal{F}$ is given by the independent sets (bases) in the intersection of two matroids $M_{1}=\left(E, \mathcal{I}_{1}\right)$ and $M_{2}=\left(E, \mathcal{I}_{2}\right)$.

All the problems above are polynomial-time solvable (see, e.g., [24]) in their unbudgeted version $(k=0)$, but become NP-hard $[1,3,7]$ even for a single budget constraint $(k=1)$. For the case of one budget $(k=1)$, polynomial-time approximation schemes (PTASs) are known for SPANNING TREE [21] (see also [11]), SHORTEST PATH [25] (see also [10,14]), and MATChing [3]. The approach in [21] easily generalizes to the case of MATROID BASIS. A PTAS is also known for MATROID INTERSECTION INDEPENDENT SET [3]. No approximation algorithm is known for the problems above in the case $k \geq 2$ (excluding multi-criteria algorithms which provide slightly infeasible solutions): investigating the existence of such algorithms is the main goal of this paper.

\subsection{Our Results}

We start by observing that several of the mentioned problems are inapproximable already for two budget constraints. More precisely, the corresponding feasibility problem is NP-complete. Due to space constraints, we omit the simple proof of the following theorem (which might be considered as part of folklore).

\footnotetext{
3 The assumption that $k$ is a constant is crucial in this paper.

${ }^{4}$ We recall that $E$ is a finite ground set and $\mathcal{I} \subseteq 2^{E}$ is a nonempty family of subsets of $E$ (independent sets) which have to satisfy the following two conditions: (i) $I \in$ $\mathcal{I}, J \subseteq I \Rightarrow J \in \mathcal{I}$ and (ii) $I, J \in \mathcal{I},|I|>|J| \Rightarrow \exists z \in I \backslash J: J \cup\{z\} \in \mathcal{I}$. A basis is a maximal independent set. For all matroids used in this paper we make the usual assumption that independence of a set can be checked in polynomial time. For additional information on matroids, see e.g. [24].
} 
Theorem 1. For $k \geq 2$, it is NP-complete to decide whether there is a feasible solution for $k$-BUDGETED SHORTEST PATH, $k$-BUDGETED PERFECT MATCHING and $k$-BUDGETED SPANNING TREE (and hence also for $k$-budgeted matroid basis and matroid intersection basis).

The remaining problems in the above list have a common aspect: the set of solutions $\mathcal{F}$ forms an independence system. In other terms, for $S \in \mathcal{F}$ and $S^{\prime} \subseteq S$, we have $S^{\prime} \in \mathcal{F}$. For these problems, we present deterministic and randomized approximation schemes, based on a variety of techniques.

Our first result is a simple but powerful mechanism to transform a multicriteria PTAS, i.e., a PTAS that might violate the budgets by a small multiplicative factor, into a pure PTAS, where no budget is violated. Similarly, a multi-criteria polynomial randomized-time approximation scheme (PRAS) can be transformed into a pure PRAS (see Section 2).

Theorem 2. (Feasibilization) Let $\mathcal{P}_{\text {ind }}$ be a $k$-budgeted problem where the set of solutions $\mathcal{F}$ is an independence system. Suppose that we are given an algorithm $\mathcal{A}$ which, for any constant $\delta>0$, computes in polynomial time an $(1-\delta)$ (resp., expected $(1-\delta)$ ) approximate solution to $\mathcal{P}_{\text {ind }}$ violating each budget by a factor at most $(1+\delta)$. Then there is a PTAS (resp., PRAS) for $\mathcal{P}_{\text {ind }}$.

The basic idea is as follows. We show that a good solution exists even if we scale down the budgets by a small factor. This is done by applying a greedy discarding strategy similar to the greedy algorithm for KNAPSACK. Applying a multi-criteria PTAS (given as a black box) to the scaled problem gives a feasible solution for the original one, of weight close to the optimal weight.

To the best of our knowledge, this simple result was never observed before. Indeed, it implies improved approximation algorithms for a number of problems. A general construction by Papadimitriou and Yannakakis [18] provides multi-criteria PTASs (resp., PRASs) for problems whose exact version admits a pseudo-polynomial-time (PPT) deterministic (resp., Monte-Carlo) algorithm. We recall that the exact version of a given optimization problem asks for a feasible solution of exactly a given target weight. Combining their approach with our mechanism one obtains approximation schemes for several problems. For example, using the PPT-algorithm for EXACT FOREST in [2], one obtains a PTAS for $k$-BUdGeted Forest. Similarly, the Monte-Carlo PPT-algorithm for EXACT MATChing in [17] gives a PRAS for $k$-BUdgeted matching. The Monte-Carlo PPT-algorithms for EXACT MATROID INTERSECTION INDEPENDENT SET in [5], which works in the special case of representable matroids ${ }^{5}$, implies a PRAS for the corresponding budgeted problem.

Of course, one can also exploit multi-criteria approximation schemes obtained with different techniques. For example, exploiting the multi-criteria PTAS in [8] for $k$-BUDGETED MATCHING in bipartite graphs, which is based on iterative rounding, one obtains a PTAS for the same problem. Very recently [6], a

\footnotetext{
${ }^{5}$ A matroid $M=(E, \mathcal{I})$ is representable if its ground set $E$ can be mapped in a bijective way to the columns of a matrix over some field, and $I \subseteq E$ is independent in $M$ iff the corresponding columns are linearly independent.
} 
multi-criteria PRAS for $k$-BUDGETED MATROID INDEPENDENT SET, based on dependent randomized rounding, has been presented. This implies a PRAS for $k$-BUDGETED MATROID INDEPENDENT SET.

Corollary 1. There are PTASs for $k$-BUDGETED FOREST and $k$-BUDGETED MATCHING in bipartite graphs. There are PRASs for $k$-BUDGETED MATCHING, $k$-BUDGETED MATROID INDEPENDENT SET, and $k$-BUDGETED MATROID INTERSECTION in representable matroids.

Based on a different, more direct approach, we are able to turn the PRAS for $k$-BUDGETED MATROID INDEPENDENT SET into a PTAS. The main insight is the following structural property of faces of the matroid polytope which might be of independent interest (proof in Section 3).

Theorem 3. Let $M=(E, \mathcal{I})$ be a matroid and let $F$ be a face of dimension $d$ of the matroid polytope ${ }^{6} P_{\mathcal{I}}$. Then any $x \in F$ has at most $2 d$ non-integral components. Furthermore, the sum of all fractional components of $x$ is at most $d$.

A PTAS can then be easily derived as follows. We first guess the $k / \varepsilon$ elements $E_{H}$ of largest weight in the optimum solution in a preliminary phase, and reduce the problem consequently. This guessing step guarantees that the maximum weight $\mathrm{w}_{\max }$ of an element in the reduced problem satisfies $k \mathrm{w}_{\max } \leq \varepsilon \mathrm{w}\left(E_{H}\right)$. For the reduced problem, we compute an optimal fractional vertex solution $x^{*}$ to the LP which seeks to find a maximum weight point in the matroid polytope intersected with the $k$ budget constraints. Since $x^{*}$ is chosen to be a vertex solution, and only $k$ linear constraints are added to the matroid polytope, $x^{*}$ lies on a face of the matroid polytope of dimension at most $k$. We then round down the fractional components of $x^{*}$ to obtain an incidence vector $\bar{x}$ which corresponds to some independent set $E_{L}$. By Theorem $3,\left|x^{*}-\bar{x}\right| \leq k$, and hence, $\mathrm{w}\left(E_{L}\right) \geq \mathrm{w}\left(x^{*}\right)-k \mathrm{w}_{\max }$. Then, it is not hard to see that $E_{H} \cup E_{L}$ is a $(1-\varepsilon)$-approximate feasible solution for the starting problem.

Corollary 2. There is a PTAS for $k$-BUDGETED MATROID INDEPENDENT SET.

Eventually, we present a PTAS (rather than a PRAS as in Corollary 1) for 2-BUdGeted Matching (see Section 4).

Theorem 4. There is a PTAS for 2-BUDGETED MATCHING.

Our PTAS works as follows. Let us confuse a matching $M$ with the associated incidence vector $x_{M}$. We initially compute an optimal fractional matching $x^{*}$, and express it as the convex combination $x^{*}=\alpha_{1} x_{1}+\alpha_{2} x_{2}+\alpha_{3} x_{3}$ of three matchings $x_{1}, x_{2}$, and $x_{3}$. Then we exploit a patching procedure which, given two matchings $x^{\prime}$ and $x^{\prime \prime}$ with high Lagrangian weight and a parameter $\mu \in[0,1]$, computes a matching $z$ which is not longer than $x_{\mu}:=\mu x^{\prime}+(1-\mu) x^{\prime \prime}$ with respect to both lengths, and has a comparable weight. This procedure is applied

\footnotetext{
${ }^{6}$ For some given matroid $M=(E, \mathcal{I})$, the corresponding matroid polytope $P_{\mathcal{I}}$ is the
} convex hull of the incidence vectors of all independent sets. 
twice: first on the matchings $x_{1}$ and $x_{2}$ with parameter $\mu=\alpha_{1} /\left(\alpha_{1}+\alpha_{2}\right)$, hence getting a matching $z^{\prime}$. Second, on the two matchings $z^{\prime}$ and $x_{3}$ with parameter $\mu=\left(\alpha_{1}+\alpha_{2}\right) /\left(\alpha_{1}+\alpha_{2}+\alpha_{3}\right)$. The resulting matching $z^{\prime \prime}$ is feasible and almost optimal (modulo a preliminary guessing step).

Our patching procedure relies on a topological property of curves in $\mathbb{R}^{2}$, that we prove via Jordan's curve theorem [15]. An extension of the property above to curves in $\mathbb{R}^{k}$ would imply a PTAS for $k$-BUDGETED MATCHING: this is left as an interesting open problem (details are omitted for lack of space).

\subsection{Related Work}

There are a few general tools for designing approximation algorithms for budgeted problems. One basic approach is combining dynamic programming (which solves the problem for polynomial weights and lengths) with rounding and scaling techniques (to reduce the problem to the case of polynomial quantities). This leads for example to the FPTAS for 1-BUDGETED SHORTEST PATH $[10,14,25]$. Another fundamental technique is the Lagrangian relaxation method. The basic idea is relaxing the budget constraints, and lifting them into the objective function, where they are weighted by Lagrangian multipliers. Solving the relaxed problem, one obtains two or more solutions with optimal Lagrangian weight, which can - if needed - be patched together to get a good solution for the original problem. Demonstrating this method, Goemans and Ravi [21] gave a PTAS for 1-BUDGETED SPANNING TREE, which also extends to 1-BUDGETED MATROID BASIS. Using the same approach, with an involved patching step, Berger, Bonifaci, Grandoni, and Schäfer [3] obtained a PTAS for 1-BUdGETEd MATCHInG and 1-BUdGeted MATROID INTERSECTION INDEPENDENT SET. Their approach does not seem to generalize to the case of multiple budget constraints.

The techniques above apply to the case of one budget. Not much is known for problems with two or more budgets. However, often multi-criteria approximation schemes are known, which provide a $(1-\varepsilon)$-approximate solution violating the budgets by a factor $(1+\varepsilon)$. First of all, there is a very general technique by Papadimitriou and Yannakakis [18], based on the construction of $\varepsilon$-approximate Pareto curves. Given an optimization problem with multiple objectives, the Pareto curve consists of the set of solutions $S$ such that there is no solution $S^{\prime}$ which is strictly better than $S$ (in a vectorial sense). Papadimitriou and Yannakakis show that, for any constant $\varepsilon>0$, there always exists a polynomial-size $\varepsilon$-approximate Pareto curve $\mathcal{A}$, i.e., a set of solutions such that every solution in the Pareto curve is within a factor of $(1+\varepsilon)$ from some solution in $\mathcal{A}$ on each objective. Furthermore, this approximate curve can be constructed in polynomial time in the size of the input and $1 / \varepsilon$ whenever there exists a PPT algorithm for the associated exact problem. This implies multi-criteria FPTASs for $k$-BUdGETED SPANNING TREe and $k$-BUdGeted SHORTEST PATH. Furthermore, it implies a multi-criteria FPRAS for $k$-BUDGETED (PERFECT) MATCHING. The latter result exploits the Monte-Carlo PPT algorithm for EXACT MATCHING in [17]. Our PRAS improves on these results, approximation-wise (the running time is larger in our case). 
Recently, Grandoni, Ravi and Singh [8] showed that the iterative rounding technique is an alternative way to achieve similar (or better) results. Using this method they obtain a multi-criteria PTAS for $k$-BUDGETED SPANNING TREE, which computes a solution of optimal cost violating each budget by a factor $(1+\varepsilon)$. This improves, approximation-wise, on the result in [18] for the same problem (where the solution returned is suboptimal). The authors also show how to obtain a deterministic (rather than randomized [18]) multi-criteria PTAS for $k$-BUDGETED MATCHING in bipartite graphs.

All mentioned problems are easy in the unbudgeted version. Given an NPhard unbudgeted problem which admits a $\rho$ approximation, the parametric search technique in [16] provides a multi-criteria $k \rho$ approximation algorithm violating each budget by a factor $k \rho$ for the corresponding problem with $k$ budgets. Other techniques lead to logarithmic approximation factors (see, e.g., $[4,19,20]$ ).

\section{A Feasibilization Mechanism}

Proof (Theorem 2). Let $\varepsilon \in(0,1]$ be a given constant, with $1 / \varepsilon \in \mathbb{N}$. Consider the following algorithm. Initially we guess the $h=k / \varepsilon$ elements $E_{H}$ of $O P T$ of largest weight, and reduce the problem consequently ${ }^{7}$, hence getting a problem $\mathcal{P}^{\prime}$. Then we scale down all the budgets by a factor $(1-\delta)$, and solve the resulting problem $\mathcal{P}^{\prime \prime}$ by means of $\mathcal{A}$, where $\delta=\varepsilon /(k+1)$. Let $E_{L}$ be the solution returned by $\mathcal{A}$. We finally output $E_{H} \cup E_{L}$.

Let $O P T^{\prime}$ and $O P T^{\prime \prime}$ be the optimum solution to problems $\mathcal{P}^{\prime}$ and $\mathcal{P}^{\prime \prime}$, respectively. We also denote by $\mathrm{L}_{i}^{\prime}$ and $\mathrm{L}_{i}^{\prime \prime}$ the $i$ th budget in the two problems, respectively. Let $\mathrm{w}_{\max }$ be the largest weight in $\mathcal{P}^{\prime}$ and $\mathcal{P}^{\prime \prime}$. We observe that trivially: (a) $\mathrm{w}(O P T)=\mathrm{w}\left(E_{H}\right)+\mathrm{w}\left(O P T^{\prime}\right)$ and $(\mathrm{b}) \mathrm{w}_{\max } \leq \mathrm{w}\left(E_{H}\right) / h$.

Let us show that $(\mathrm{c}) \mathrm{w}\left(O P T^{\prime \prime}\right) \geq \mathrm{w}\left(O P T^{\prime}\right)(1-k \delta)-k \mathrm{w}_{\max }$. Consider the following process: for each length function $i$, we remove from $O P T^{\prime}$ the element $e$ with smallest ratio $\mathrm{w}(e) / \ell_{i}(e)$ until $\ell_{i}\left(O P T^{\prime}\right) \leq(1-\delta) \mathrm{L}_{i}^{\prime}$. Let $E_{i}$ be the set of elements removed. It is not hard to see that $\mathrm{w}\left(E_{i}\right) \leq \delta \mathrm{w}\left(O P T^{\prime}\right)+\mathrm{w}_{\max }$. It follows that $O P T^{\prime}-\cup_{i} E_{i}$ is a feasible solution for $\mathcal{P}^{\prime \prime}$ of weight at least $w\left(O P T^{\prime}\right)(1-\delta k)-k \mathrm{w}_{\max }$, proving (c).

We observe that $E_{L}$ is feasible for $\mathcal{P}^{\prime}$ since, for each $i, \ell_{i}\left(E_{L}\right) \leq(1+\delta) L_{i}^{\prime \prime}=$ $(1+\delta)(1-\delta) L_{i}^{\prime} \leq L_{i}^{\prime}$. As a consequence, the returned solution $E_{H} \cup E_{L}$ is feasible. Moreover, when $\mathcal{A}$ is deterministic, we have

$$
\begin{aligned}
w\left(E_{H}\right)+w\left(E_{L}\right) & \geq w\left(E_{H}\right)+(1-\delta) w\left(O P T^{\prime \prime}\right) \\
& \stackrel{(c)}{\geq} w\left(E_{H}\right)+(1-\delta)\left(w\left(O P T^{\prime}\right)(1-\delta k)-k \mathrm{w}_{\max }\right) \\
& \stackrel{(b)}{\geq}(1-k / h) w\left(E_{H}\right)+(1-\delta(k+1)) w\left(O P T^{\prime}\right) \\
& \geq(1-\varepsilon)\left(w\left(E_{H}\right)+w\left(O P T^{\prime}\right)\right) \stackrel{(a)}{=}(1-\varepsilon) w(O P T) .
\end{aligned}
$$

The same bound holds in expectation when $\mathcal{A}$ is randomized.

\footnotetext{
7 As usual, by reducing we mean decreasing each budget $\mathrm{L}_{i}$ by $\ell_{i}\left(E_{H}\right)$ and removing all the elements of weight strictly larger than $\min _{e \in E_{H}} \mathrm{w}(e)$. By guessing we mean trying all the $O\left(m^{h}\right)$ subsets of $h$ elements.
} 


\section{A PTAS for $\boldsymbol{k}$-Budgeted Matroid Independent Set}

It is convenient to consider weights $\mathrm{w}$ and lengths $\ell_{i}$ as vectors in $\mathbb{Q}^{E}$. We denote by $\ell$ the matrix whose $i$ th column is $\ell_{i}$, and let $L=\left(L_{1}, \ldots, L_{k}\right)^{T}$. A rank function $r: 2^{E} \rightarrow \mathbb{N}$ is associated to every matroid $M=(E, \mathcal{I})$; it is defined by $r(S)=\max \{|J| \mid J \subseteq S, J \in \mathcal{I}\}$. The matroid polytope $P_{\mathcal{I}}$ is the convex hull of the characteristic vectors $\chi_{I}$ of the independent sets $I \in \mathcal{I}$ and is described by the following set of inequalities: $P_{\mathcal{I}}=\operatorname{conv}\left\{\chi_{I}: I \in \mathcal{I}\right\}=\{x \geq 0: x(S) \leq$ $r(S) \forall S \subseteq E$. As usual, $x(S):=\sum_{e \in S} x(e)^{8}$.

Proof (Theorem 3). Let $m=|E|$. We assume that the matroid polytope has full dimension, i.e., $\operatorname{dim}\left(P_{\mathcal{I}}\right)=m$, or equivalently, every element $e \in E$ is independent. This can be assumed wlog since if $\{e\} \notin \mathcal{I}$ for some $e \in E$, then we can reduce the matroid by deleting element $e$. Since $\operatorname{dim}\left(P_{\mathcal{I}}\right)=m$ and $\operatorname{dim}(F)=d$, $F$ can be described by the inequality system of $P_{\mathcal{I}}$, where $m-d$ linearly independent inequalities used in the description of $P_{\mathcal{I}}$ are turned into equalities. More precisely, there are $N \subseteq E$ and $A_{1}, \ldots, A_{k} \subseteq E$ such that

$$
F=\left\{x \in P_{\mathcal{I}} \mid x(e)=0 \forall e \in N, x\left(A_{i}\right)=r\left(A_{i}\right) \forall i \in\{1, \ldots, k\}\right\},
$$

and $|N|+k=m-d$. By standard uncrossing arguments, we can assume that the sets $A_{i}$ form a chain, i.e., $A_{1} \subsetneq A_{2} \subsetneq \cdots \subsetneq A_{k}$ (see for example [9, 12] for further information on combinatorial uncrossing). We prove the claim by induction on the number of elements of the matroid. The theorem clearly holds for matroids with a ground set of cardinality one. First assume $N \neq \emptyset$ and let $e \in N$. Let $M^{\prime}$ be the matroid obtained from $M$ by deleting $e$, and let $F^{\prime}$ be the projection of $F$ onto the coordinates corresponding to $N \backslash\{e\}$. Since $F^{\prime}$ is a face of $M^{\prime}$, the claim follows by induction. Henceforth, we assume $N=\emptyset$ which implies $k=m-d$. Let $A_{0}=\emptyset$ and $B_{i}=A_{i} \backslash A_{i-1}$ for $i \in\{1, \ldots, k\}$. In the following we show that we can assume

$$
0<r\left(A_{i}\right)-r\left(A_{i-1}\right)<\left|B_{i}\right| \quad \forall i \in\{1, \ldots, k\} .
$$

Notice that $0 \leq r\left(A_{i}\right)-r\left(A_{i-1}\right) \leq\left|B_{i}\right|$ clearly holds by standard properties of rank functions (see [24] for more details). Assume that there is $i \in\{1, \ldots, k\}$ with $r\left(A_{i}\right)=r\left(A_{i-1}\right)$. Since all points $x \in F$ satisfy $x\left(A_{i}\right)=r\left(A_{i}\right)$ and $x\left(A_{i-1}\right)=$ $r\left(A_{i-1}\right)$, we have $x\left(B_{i}\right)=0$. Hence for any $e \in B_{i}$, we have $x(e)=0$ for $x \in F$. Again, we can delete $e$ from the matroid, hence obtaining a smaller matroid for which the claim holds by the inductive hypothesis. Therefore, we can assume $r\left(A_{i}\right)>r\left(A_{i-1}\right)$ which implies the left inequality in (1).

For the right inequality assume that there is $i \in\{1, \ldots, k\}$ with $r\left(A_{i}\right)-$ $r\left(A_{i-1}\right)=\left|B_{i}\right|$. Hence, every $x \in F$ satisfies $x\left(B_{i}\right)=\left|B_{i}\right|$, implying $x(e)=1$ for all $e \in B_{i}$. Let $e \in B_{i}$, and let $F^{\prime}$ be the projection of the face $F$ onto the components $N \backslash\{e\}$. Since $F^{\prime}$ is a face of the matroid $M^{\prime}$ obtained from $M$ by contracting $e$, the result follows again by the inductive hypothesis.

Henceforth, we assume that (1) holds. This implies in particular that $\left|B_{i}\right|>1$ for $i \in\{1, \ldots, k\}$. Since $\sum_{i=1}^{k}\left|B_{i}\right| \leq m$, we have $k \leq m / 2$, which together with

\footnotetext{
${ }^{8}$ See $[24]$ for more details and omitted standard definitions.
} 
$k=m-d$ implies $d \geq m / 2$. The claim of the theorem that $x \in F$ has at most $2 d$ non-integral components is thus trivial in this case.

To prove the second part of the theorem we show that if (1) holds then $x(E) \leq d$ for $x \in F$. For $x \in F$ we have

$$
\begin{aligned}
x(E) & =x\left(E \backslash A_{k}\right)+\sum_{i=1}^{k} x\left(B_{i}\right) \leq|E|-\left|A_{k}\right|+\sum_{i=1}^{k}\left(r\left(A_{i}\right)-r\left(A_{i-1}\right)\right) \\
& \leq|E|-\left|A_{k}\right|+\sum_{i=1}^{k}\left(\left|A_{i}\right|-\left|A_{i-1}\right|-1\right)=m-k=d,
\end{aligned}
$$

where the first inequality follows from $x\left(E \backslash A_{k}\right) \leq\left|E \backslash A_{k}\right|$ and $x\left(B_{i}\right)=r\left(A_{i}\right)-$ $r\left(A_{i-1}\right)$, and the second inequality follows from (1).

\section{A PTAS for 2-BUDGETED MATCHING}

In this section we present our PTAS for 2-BUDGETED MATCHING. We denote by $\mathcal{M}$ the set of incidence vectors of matchings. With a slight abuse of terminology we call the elements in $\mathcal{M}$ matchings. Let $P_{\mathcal{M}}$ be the matching polyhedron. Analogously to Section 3 , let $\ell=\left(\ell_{1}, \ell_{2}\right)$ and $\mathrm{L}=\left(\mathrm{L}_{1}, \mathrm{~L}_{2}\right)^{T}$. A feasible solution in this framework is a matching $x \in \mathcal{M}$ such that $\ell^{T} x \leq \mathrm{L}$. For two elements $z^{\prime}, z^{\prime \prime} \in$ $[0,1]^{E}$, we define their symmetric difference $z^{\prime} \Delta z^{\prime \prime} \in[0,1]^{E}$ by $\left(z^{\prime} \Delta z^{\prime \prime}\right)(e)=$ $\left|z^{\prime}(e)-z^{\prime \prime}(e)\right|$ for all $e \in E$. In particular, if $z^{\prime}$ and $z^{\prime \prime}$ are incidence vectors, then their symmetric difference as defined above corresponds indeed to the symmetric difference in the usual sense. Recall that, when $z^{\prime}$ and $z^{\prime \prime}$ are matchings, $z^{\prime} \Delta z^{\prime \prime}$ consists of a set of node-disjoint paths and cycles.

We start by presenting a property of curves in $\mathbb{R}^{2}$. This property is used to derive the mentioned patching procedure. Eventually, we describe and analyze our PTAS.

A Property of Curves in $\mathbb{R}^{2}$. We next describe a topological property of polygonal curves in $\mathbb{R}^{2}$, which will be crucial in our proof ${ }^{9}$. A curve in $\mathbb{R}^{2}$ is a continuous function $f:[0, \tau] \rightarrow \mathbb{R}^{2}$ for some $\tau \in \mathbb{R}_{+}$. A curve is called polygonal if it is piecewise linear. For $a \in[0, \tau]$, let $f^{a}:[0, \tau] \rightarrow \mathbb{R}^{2}$ be the following curve.

$$
f^{a}(t)= \begin{cases}f(t+a)-f(a)+f(0) & \text { if } t+a<\tau, \\ f(\tau)-f(a)+f(a+t-\tau) & \text { if } t+a \geq \tau .\end{cases}
$$

Observe that $f^{a}(0)=f(0)$ and $f^{a}(\tau)=f(\tau)$ for any $a \in[0, \tau]$. The next lemma shows that any point $x$ on the segment between $f(0)$ and $f(\tau)$ is contained in some curve $f^{a}$.

Lemma 1. Let $f:[0, \tau] \rightarrow \mathbb{R}^{2}$ be a polygonal curve, and let $\mu \in[0,1]$. Then there are $a, t \in[0, \tau]$ such that $f^{a}(t)=\mu f(0)+(1-\mu) f(\tau)$.

We next give an intuitive description of the proof of the lemma: a formal proof is postponed to the journal version of the paper. Let $f=\left(f_{1}, f_{2}\right)$. Since the statement of the lemma is independent of changes in the coordinate system

\footnotetext{
9 The lemma even holds for general (non-polygonal) curves. However, since we only need polygonal curves in our setting we restrict ourselves to this case since it simplifies the exposition.
} 
(and the claim is trivial for $f(0)=f(\tau)$ ), we can assume that $f(0)=(0,0)$ and $f(\tau)=(r, 0)$ for some $r>0$. The Gasoline Lemma [3] states that there is $a_{1} \in[0, \tau]$ such that $f_{2}^{a_{1}}(t) \geq 0 \forall t \in[0, \tau]$. In particular, this condition is satisfied by choosing $a_{1} \in \arg \min \left\{f_{2}(t) \mid t \in[0, \tau]\right\}$. Analogously, for $a_{2} \in$ $\arg \max \left\{f_{2}(t) \mid t \in[0, \tau]\right\}, f_{2}^{a_{2}}(t) \leq 0 \forall t \in[0, \tau]$. Hence, we have two curves, $f^{a_{1}}$ and $f^{a_{2}}$, one above and the other below the $\mathrm{x}$-axis, both with the same endpoints $(0,0)$ and $(r, 0)$. Furthermore, for $a$ ranging from $a_{1}$ to $a_{2}$ (in a circular sense), the curve $f^{a}$ continuously transforms from $f^{a_{1}}$ to $f^{a_{2}}$, always maintaining the same endpoints. Then it is intuitively clear that the union of the curves $f^{a}$ spans all the points on the segment from $(0,0)$ to $(r, 0)$, hence proving the claim.

The Patching Procedure. In this section we describe a patching procedure which, given two matchings $x^{\prime}$ and $x^{\prime \prime}$ and a parameter $\mu \in[0,1]$, computes a matching $z$ satisfying $\ell^{T} z \leq \ell^{T} x_{\mu}$, where $x_{\mu}:=\mu x^{\prime}+(1-\mu) x^{\prime \prime}$ is a convex combination of the first two matchings. Furthermore, the weight $\mathrm{w}^{T} z$ is close to $\mathrm{w}^{T} x_{\mu}$, provided that $x^{\prime}$ and $x^{\prime \prime}$ have a sufficiently large Lagrangian weight, which is defined as follows. Let $\lambda_{1}^{*}, \lambda_{2}^{*} \in \mathbb{R}_{+}$be a pair of optimal dual multipliers for the budgets in the linear program $\max \left\{\mathrm{w}^{T} x \mid x \in P_{\mathcal{M}}, \ell^{T} x \leq L\right\}$. The Langrangian weight of $x \in[0,1]^{E}$ is $\mathcal{L}(x)=\mathrm{w}^{T} x-\left(\lambda_{1}^{*}, \lambda_{2}^{*}\right)\left(\ell^{T} x-\mathrm{L}\right)$. Notice, that by the theory of Lagrangian duality we have $\mathrm{w}^{*}=\max \left\{\mathcal{L}(x) \mid x \in P_{\mathcal{M}}\right\}$, where $\mathrm{w}^{*}$ is the weight of an optimal LP solution, i.e., $\mathrm{w}^{*}=\max \left\{\mathrm{w}^{T} x \mid x \in P_{\mathcal{M}}, \ell^{T} x \leq L\right\}$ (see [13] for more information on Lagrangian duality).

We need the following notion of almost matching.

Definition 1. For $r \in \mathbb{N}$, an $r$-almost matching in $G$ is a (possibly fractional) vector $y \in[0,1]^{E}$ such that it is possible to set at most $r$ components of $y$ to zero to obtain a matching.

We denote by $\mathcal{M}_{r}$ the set of all $r$-almost matchings in $G$. Given an $r$-almost matching $y$, we let a corresponding matching $z \in \mathcal{M}$ be a matching obtained by setting to zero the fractional components of $y$, and then computing a maximal matching in the resulting set of edges (in particular, we might need to set to 0 some 1 entries of $y$ to obtain $z$ ). Notice that $\mathrm{w}^{T} z \geq \mathrm{w}^{T} y-r \mathrm{w}_{\max }$, where $w_{\max }$ is the largest weight.

Our patching procedure first constructs a 2-almost matching $y$, and then returns a corresponding matching $z$. We next show how to compute $y$. Let us restrict our attention to the following set of candidate 2-almost matchings. Recall that $s=x^{\prime} \Delta x^{\prime \prime}$ is a set of paths and cycles. We construct an auxiliary graph $C$, consisting of one cycle $\left(e_{0}, e_{1}, \ldots, e_{\tau-1}\right)$, with the following property: there is a bijective mapping between the edges of $C$ and the edges of $s$ such that two consecutive edges of $C$ are either consecutive in some path/cycle or belong to different paths/cycles. This can be easily achieved by cutting each cycle, appending the resulting set of paths one to the other, and gluing together the endpoints of the obtained path. For $t \in[0, \tau]$, we define $s(t) \in[0,1]^{E}$ as

$$
(s(t))(e)= \begin{cases}1 & \text { if } e=e_{i}, i<\lfloor t\rfloor \\ t-\lfloor t\rfloor & \text { if } e=e_{i}, i=\lfloor t\rfloor \\ 0 & \text { otherwise. }\end{cases}
$$


Moreover, for $a, t \in[0, \tau]$, we define

$$
[0,1]^{E} \ni s^{a}(t)= \begin{cases}s(a+t)-s(a) & \text { if } a+t \leq \tau ; \\ s(a+t-\tau)+s(\tau)-s(a) & \text { if } a+t>\tau .\end{cases}
$$

Intuitively, $a$ and $(a+t)(\bmod \tau)$ define a (fractional) subpath of $C$, and $s^{a}(t)$ is the (fractional) incidence vector corresponding to that subpath. Additionally we define $y^{a}(t):=x^{\prime} \triangle s^{a}(t)$. Note that $y^{a}(t)$ is equal to $x^{\prime}$ and $x^{\prime \prime}$ for $t=0$ and $t=\tau$, respectively.

Lemma 2. For any $a, t \in[0, \tau], y^{a}(t)$ is a 2-almost matching.

Proof. One can easily observe that a matching can be obtained by setting the two components of $y^{a}(t)$ to zero that correspond to the edges $e_{\lfloor a\rfloor}$ and $e_{\lfloor(a+t)(\bmod \tau)\rfloor}$.

The following lemma shows that, in polynomial time, one can find a 2-almost matching $y$ with lengths $\ell^{T} y$ equal to the lengths of any convex combination of the two matchings $x^{\prime}$ and $x^{\prime \prime}$.

Lemma 3. Let $\mu \in[0,1]$ and $x_{\mu}=\mu x^{\prime}+(1-\mu) x^{\prime \prime}$. In polynomial time, $a, t \in$ $[0, \tau]$ can be determined such that $\ell^{T} y^{a}(t)=\ell^{T} x_{\mu}$.

Proof. Let $f:[0, \tau] \rightarrow \mathbb{R}^{2}$ be the polygonal curve defined by $f(t)=\ell^{T} y^{0}(t)$. Since $f(0)=\ell^{T} x^{\prime}$ and $f(\tau)=\ell^{T} x^{\prime \prime}$, we have by Lemma 1 that there exists $a, t \in[0, \tau]$ such that $f^{a}(t)=\ell^{T} x_{\mu}$. Since $f^{a}(t)=\ell^{T} y^{a}(t), y:=y^{a}(t)$ satisfies the claim. The values of $\lfloor a\rfloor$ and $\lfloor a+t\rfloor$ can be guessed in polynomial time by considering $O\left(n^{2}\right)$ possibilities. Given those two rounded values, the actual values of $a$ and $t$ can be obtained by solving a linear program with a constant number of variables and constraints.

Our patching procedure computes a 2-almost matching $y=y^{a}(t)$ with $\ell^{T} y=$ $\ell^{T} x_{\mu}$, exploiting the lemma above, and then returns a corresponding matching $z$, by applying the procedure explained in the proof of Lemma 2 . Trivially, $\ell^{T} z \leq$ $\ell^{T} y=\ell^{T} x_{\mu}$. We next show that, if $x^{\prime}$ and $x^{\prime \prime}$ have sufficiently large Lagrangian weight, then the weight of $z$ is close to the weight of $x_{\mu}$.

Lemma 4. Assume $\mathcal{L}\left(x^{\prime}\right) \geq \mathrm{w}^{*}-\Gamma$ and $\mathcal{L}\left(x^{\prime \prime}\right) \geq \mathrm{w}^{*}-\Gamma$ for some $\Gamma \in \mathbb{R}_{+}$. Then the matching $z$ returned by the patching procedure satisfies $\mathrm{w}^{T} z \geq \mathrm{w}^{T} x_{\mu}-$ $2 \mathrm{w}_{\max }-\Gamma$ and $\ell^{T} z \leq \ell^{T} x_{\mu}$.

Proof. By Lemma 3 we have $\ell^{T} y=\ell^{T} x_{\mu}$, and since $z \leq y$, we get $\ell^{T} z \leq \ell^{T} x_{\mu}$. Let $\bar{x}_{\mu}=x^{\prime}+x^{\prime \prime}-x_{\mu}=(1-\mu) x^{\prime}+\mu x^{\prime \prime}$. Since $\mathcal{L}\left(x^{\prime}\right) \geq \mathrm{w}^{*}-\Gamma, \mathcal{L}\left(x^{\prime \prime}\right) \geq \mathrm{w}^{*}-\Gamma$ and $\mathcal{L}$ is linear, we have $\mathcal{L}\left(x_{\mu}\right) \geq \mathrm{w}^{*}-\Gamma$ and $\mathcal{L}\left(\bar{x}_{\mu}\right) \geq \mathrm{w}^{*}-\Gamma$. Recall that $y=y^{a}(t)$ for a proper choice of $a, t \in[0, \tau]$. Let $\bar{y}:=x^{\prime}+x^{\prime \prime}-y$. Notice that $\bar{y}=y^{a^{\prime}}(\tau-t)$ where $a^{\prime}=(a+t)(\bmod \tau)$, and hence, $\bar{y}$ is also a 2-almost matching by Lemma 2 . Let $\bar{z}$ be the matching corresponding to $\bar{y}$ obtained by applying the procedure explained in the proof of Lemma 2 to $\bar{y}$. Notice that the pairs $(z, y)$ and $(\bar{z}, \bar{y})$ differ on the same two (or less) components. Hence

$$
\mathrm{w}^{T} z+\mathrm{w}^{T} \bar{z}+2 \mathrm{w}_{\max } \geq \mathrm{w}^{T} y+\mathrm{w}^{T} \bar{y}=\mathrm{w}^{T} x_{\mu}+\mathrm{w}^{T} \bar{x}_{\mu} .
$$


Since $y+\bar{y}=x_{\mu}+\bar{x}_{\mu}$ and $\ell^{T} y=\ell^{T} x_{\mu}$, we get $\ell^{T} \bar{y}=\ell^{T} \bar{x}_{\mu}$. Thus, $\ell^{T} \bar{z} \leq \ell^{T} \bar{x}_{\mu}$ since $\bar{z} \leq \bar{y}$. This can be rewritten as $\mathcal{L}(\bar{z})-\mathrm{w}^{T} \bar{z} \geq \mathcal{L}\left(\bar{x}_{\mu}\right)-\mathrm{w}^{T} \bar{x}_{\mu}$. Since $\mathcal{L}\left(\bar{x}_{\mu}\right) \geq \mathrm{w}^{*}-\Gamma$ and $\mathcal{L}(\bar{z}) \leq \mathrm{w}^{*}$, we obtain $\mathrm{w}^{T} \bar{z} \leq \mathrm{w}^{T} \bar{x}_{\mu}+\Gamma$. Combining this result with (2) implies $\mathrm{w}^{T} z \geq \mathrm{w}^{T} x_{\mu}-2 \mathrm{w}_{\max }-\Gamma$.

The Algorithm. Our PTAS works as follows. First it guesses the $6 / \varepsilon$ heaviest edges $E_{H}$ in the optimum solution, and reduces the problem consequently. Then it computes a vertex $x^{*} \in P_{\mathcal{M}}$ of the polytope $\left\{x \in P_{\mathcal{M}} \mid \ell^{T} x \leq L\right\}$ of maximum weight $\mathrm{w}^{*}:=\mathrm{w}^{T} x^{*}$. As $x^{*}$ is a vertex solution of the polytope $P_{\mathcal{M}}$ with two additional constraints, it lies on a face of $P_{\mathcal{M}}$ of dimension at most two. Hence, by Carathéodory's Theorem, $x^{*}$ can be expressed as a convex combination $x^{*}=$ $\alpha_{1} x_{1}+\alpha_{2} x_{2}+\alpha_{3} x_{3}$ of three matchings $x_{1}, x_{2}, x_{3} \in P_{\mathcal{M}}$. Let $\mu^{\prime}=\alpha_{1} /\left(\alpha_{1}+\alpha_{2}\right)$ and $\mu^{\prime \prime}=\left(\alpha_{1}+\alpha_{2}\right) /\left(\alpha_{1}+\alpha_{2}+\alpha_{3}\right)$. Applying Lemma 4 to $x_{1}$ and $x_{2}$ with $\mu=\mu^{\prime}$, a matching $z^{\prime}$ is obtained. Applying Lemma 4 to $z^{\prime}$ and $x_{3}$ with $\mu=\mu^{\prime \prime}$, we obtain a matching $z^{\prime \prime}$. The algorithm returns $z^{\prime \prime}$ and $E_{H}$.

Proof (Theorem 4). Consider the algorithm above. The initial guessing can be performed in $O\left(|E|^{6 / \varepsilon}\right)$ time. Since it is possible to efficiently separate over $P_{\mathcal{M}}$, $x^{*}$ can be computed in polynomial time [24]. The same holds for the decomposition of $x^{*}$ into three matchings by standard techniques (see for example [23]). Lemma 3 implies that the patching can be done in polynomial time. Hence the proposed algorithm runs in polynomial time as claimed.

Since $\mathcal{L}\left(x^{*}\right)=\mathrm{w}^{*}$ and $\mathcal{L}(x) \leq \mathrm{w}^{*}$ for $x \in P_{\mathcal{M}}$, we get $\mathcal{L}\left(x_{1}\right)=\mathcal{L}\left(x_{2}\right)=$ $\mathcal{L}\left(x_{3}\right)=\mathrm{w}^{*}$. Let $u:=\mu^{\prime} x_{1}+\left(1-\mu^{\prime}\right) x_{2}$ and $v:=\mu^{\prime \prime} z^{\prime}+\left(1-\mu^{\prime \prime}\right) x_{3}$. By Lemma 4 , matching $z^{\prime}$ satisfies $\ell^{T} z^{\prime} \leq \ell^{T} u$ and $\mathrm{w}^{T} z^{\prime} \geq \mathrm{w}^{T} u-2 \mathrm{w}_{\max }$. Since $u$ is a convex combination of $x_{1}$ and $x_{2}$, we have $\mathcal{L}(u)=\mathrm{w}^{*}$. Furthermore, by the relations between the lengths and weight of $z^{\prime}$ and $u$, we get $\mathcal{L}\left(z^{\prime}\right) \geq \mathcal{L}(u)-2 \mathrm{w}_{\max }=$ $\mathrm{w}^{*}-2 \mathrm{w}_{\max }$.

By Lemma 4, matching $z^{\prime \prime}$ satisfies $\ell^{T} z^{\prime \prime} \leq \ell^{T} v$ and $\mathrm{w}^{T} z^{\prime \prime} \geq \mathrm{w}^{T} v-4 \mathrm{w}_{\max }$. We observe that $z^{\prime \prime}$ satisfies the budget constraints since

$$
\ell^{T} z^{\prime \prime} \leq \ell^{T} v=\ell^{T}\left(\left(\alpha_{1}+\alpha_{2}\right) z^{\prime}+\alpha_{3} x_{3}\right) \leq \ell^{T}\left(\left(\alpha_{1}+\alpha_{2}\right) u+\alpha_{3} x_{3}\right)=\ell^{T} x^{*} \leq \mathrm{L} .
$$

Furthermore,

$$
\begin{aligned}
\mathrm{w}^{T} z^{\prime \prime} & \geq \mathrm{w}^{T} v-4 \mathrm{w}_{\max }=\mathrm{w}^{T}\left(\left(\alpha_{1}+\alpha_{2}\right) z^{\prime}+\alpha_{3} x_{3}\right)-4 \mathrm{w}_{\max } \\
& \geq \mathrm{w}^{T}\left(\left(\alpha_{1}+\alpha_{2}\right) u+\alpha_{3} x_{3}\right)-6 \mathrm{w}_{\max }=\mathrm{w}^{*}-6 \mathrm{w}_{\max } .
\end{aligned}
$$

Let $O P T^{\prime}$ be an optimum solution to the reduced problem. Of course, $\mathrm{w}^{*} \geq$ $\mathrm{w}\left(O P T^{\prime}\right)$. Furthermore, the weight of the guessed edges $E_{H}$ is at least $6 / \varepsilon w_{\max }$. Since $\mathrm{w}(O P T)=\mathrm{w}\left(E_{H}\right)+\mathrm{w}\left(O P T^{\prime}\right)$, we can conclude that the solution returned by the algorithm has weight at least $\mathrm{w}\left(E_{H}\right)(1-\varepsilon)+\mathrm{w}\left(O P T^{\prime}\right) \geq(1-\varepsilon) \mathrm{w}(O P T)$.

\section{References}

1. V. Aggarwal, Y. P. Aneja, and K. P. K. Nair, Minimal spanning tree subject to a side constraint, Computers \& Operations Research, 9 (1982), pp. 287-296. 
2. F. Barahona, And W. R. Pulleyblank, Exact arborescences, matchings and cycles, Discrete Applied Mathematics, 16(2): 91-99, 1987.

3. A. Berger, V. Bonifaci, F. Grandoni, And G. Schäfer. Budgeted matching and budgeted matroid intersection via the gasoline puzzle. To appear in Mathematical Programming. Preliminary version in IPCO 2008.

4. V. Bilu, V. Goyal, R. Ravi, And M. Singh. On the crossing spanning tree problem. In APPROX-RANDOM, pages 51-64, 2004.

5. P. Camerini, G. Galbiati, And F. Maffioli, Random pseudo-polynomial algorithms for exact matroid problems, Journal of Algorithms 13: 258-273, 1992.

6. C. Chekuri, J. Vondrák, And R. Zenklusen, Dependent randomized rounding for matroid polytopes and applications, 2009. http://arxiv.org/abs/0909.4348.

7. M. R. Garey And D. S. Johnson, Computers and intractability: A guide to the theory of NP-completeness, W.H. Freeman, 1979.

8. F. Grandoni, R. Ravi, And M. Singh, Iterative rounding for multi-objective optimization problems. In ESA, pages 95-106, 2009.

9. C. A. Hurkens, L. Lovász, A. Schrijver, and E. Tardos, How to tidy up your set system, Combinatorics, North-Holland, 309-314, 1988.

10. R. HAssin, Approximation schemes for the restricted shortest path problem, Mathematics of Operation Research 17(1): 36-42, 1992.

11. R. HASSIN AND A. LEVIn, An efficient polynomial time approximation scheme for the constrained minimum spanning tree problem using matroid intersection, SIAM Journal on Computing 33(2): 261-268, 2004.

12. K. JAIN, A factor 2 approximation algorithm for the generalized Steiner network problem, Combinatorica 21: 39-60, 2001.

13. B. Korte And J. Vygen, Combinatorial optimization, Springer, 2008.

14. D. LORENZ AND D. RAZ, A simple efficient approximation scheme for the restricted shortest paths problem, Operations Research Letters 28: 213-219, 2001.

15. J. R. Munkres, Topology, second edition, Prentice Hall, 2000.

16. M. V. Marathe, R. Ravi, R. Sundaram, S. S. Ravi, D. J. Rosenkrantz, and H. B. Hunt III. Bicriteria network design problems. In ICALP, pages 487-498, 1995.

17. K. Mulmuley, U. Vazirani, And V. Vazirani, Matching is as easy as matrix inversion. Combinatorica, 7(1):101-104, 1987.

18. C. H. Papadimitriou And M. Yannakakis, On the approximability of trade-offs and optimal access of Web sources. In FOCS, pages 86-92, 2000.

19. R. RAVI. Rapid rumor ramification: Approximating the minimum broadcast time. In FOCS, pages 202-213, 1994.

20. R. RAVI. Matching based augmentations for approximating connectivity problems. Invited Lecture, In LATIN, pages 13-24, 2006.

21. R. Ravi and M. X. Goemans. The constrained minimum spanning tree problem (extended abstract). In $S W A T$, pages 66-75, 1996.

22. R. Ravi, M. V. Marathe, S. S. Ravi, D. J. Rosenkrantz, and H. B. Hunt. Many birds with one stone: Multi-objective approximation algorithms. In STOC, pages 438-447, 1993.

23. A. SchriJver, Theory of linear and integer programming, John Wiley \& Sons, 1998.

24. A. SCHRIJVER, Combinatorial optimization, polyhedra and efficiency, Springer, 2003.

25. A. Warburton, Approximation of Pareto optima in multiple-objective, shortest path problems. Operations Research, 35: 70-79, 1987. 